\title{
Sediment tracers in water erosion studies: Current approaches and challenges
}

Gema Guzmán (1), John N Quinton (2), Mark A Nearing (3), Lionel Mabit (4), Juan V Giráldez (1,5), and José A Gómez (1)

(1) Institute for Sustainable Agriculture-CSIC, Córdoba, Spain (g92gudim@uco.es, joseagomez@ias.csic.es), (2) Lancaster Environment Centre, Lancaster University, Lancaster, UK, (3) USDA-ARS, Southwest Watershed Research Center, Tucson, USA, (4) Environmental Geosciences, Department of Environmental Sciences, University of Basel, Switzerland, (5) University of Córdoba, Agronomy, Córdoba, Spain (ag1gicej@uco.es)

The quest for alternative methods of soil losses assessment, due to water erosion to complement and enhance existing methods has directed attention to the use of tracing approaches because of the additional information they provide, such as sediment source identification, tracking of sediment movement across the landscape at various temporal and spatial scales and soil erosion rates. For these reasons, the utility and robustness of sediment tracing approaches using a wide range of substances and soil properties have been evaluated in numerous studies.

A comprehensive literature review on tracing approaches used in water erosion studies was carried out in June 2011 using the Web of Science database and as search terms in the title or as keywords: "erosion AND tracer" OR "sediment AND tracer" OR "sediment AND tracking”. The search excluded reviews and tillage and/or wind erosion studies. Only studies that used tracers to make a determination of water erosion or sedimentation rates, or in some cases relative erosion contribution, were considered in this study, and were further refined by manually checking that the articles corresponded to experiments involving sediment studies using tracers, as defined within the context of this review. Five distinct groups of tracing approaches were identified: fallout radionuclides, rare earth elements, soil magnetism and magnetic substances, other tracers, and sediment fingerprinting techniques.

This abstract presents a synthesis of the current approaches of each of the tracing techniques identified in assessing soil erosion and sediment redistribution and a summary with the commonalities and differences between the approaches and identifying research gaps and future trends. 